\title{
Better research through metrology
}

\author{
We encourage our readers to consider whether principles of measurement science could have a role to play in their \\ own disciplines.
}

M ost scientists receive little or no formal training in metrology-the science of measurement-and indeed may not even be aware that this is a discipline in itself. Though its tenets are not always directly applicable to the messy multivariate world of biological research, metrology is due more attention from biologists.

Metrology formalizes the practice of making a reliable measurement. Historically, the discipline arose around the definition of measurement units, and this remains a key component. As a consequence, metrology shapes not only scientific activities but also trade, air travel, satellite communicationanything that relies on agreed-upon systems for measuring the physical world. The importance of standard units has indeed been recognized for a long time: in roughly $800 \mathrm{AD}$, Charlemagne attempted to standardize units of length, for instance, and much earlier examples can be found as well. But it was not until the late 18th century, in the wake of the French Revolution, that states began to adopt standard units of length and weight.

In the late 19th century, the Treaty of the Meter, signed in Paris by 17 member states, established the Bureau International des Poids et Mesures (BIPM). This body was at first set up to maintain standards for the meter and the kilogram and to enable the implementation of these standards internationally. Since then, its remit has been extended to additional units, the number of member states has grown to 59, and the International System of Units (SI) has been defined. These cover seven so-called base units-the second, ampere, mole, kelvin and candela, in addition to the meter and the kilogram-as well as many derived units.

But metrology goes beyond the definition of units; measurement scientists are concerned with how to make a good measurement. The metrological mindsetan attention to minute detail and to the relationship between a measurement and the true level of the measurand-is fundamental to the ability to draw believable conclusions in any domain of science, including biology. Although many biologists do consider the quality of their measurements, including tests of technical variation and method sensitivity, this is rarely tackled systematically and can vary between disciplines.

Defining the uncertainty of a measurement is a core activity of metrology. For physical measurements there are established practices for uncertainty analysis. Extending an uncertainty analysis to biological experiments is not straightforward, as there are often many variables, several of them uncontrolled and probably even unknown, that can affect the outcome. Nevertheless, the principles of uncertainty analysis could provide biologists with at least a heuristic for assessing how confident one can be in a measurement, as argued by researchers at the National Institute for Standards and Technology (NIST), the US national metrological institute, in papers published in 2014 in Nature Methods and more recently in PLoS Biology. Considering and testing sources of uncertainty in a biological measurement also gives scientists a better understanding of the variables involved.

As has often been pointed out, if one does an experiment twice and gets a different result each time, the likelihood is strong, especially in complex biological systems, that one has inadvertently done a different experiment (i.e., that some variable changed from the first to the second attempt). By paying closer attention to sources of uncertainty, biologists may be able to better interpret and possibly even learn from situations where an experiment cannot be reproduced from one context to another.

A different metrological concept, traceability, can guide thinking about biological reference samples. Metrology defines a traceable measurement as having an unbroken, documented chain of references back to an international standard. This ensures that measurements can be compared - that a third of a millimeter measured for a hypothetical Japanese aircraft part will match the third of a millimeter of its complementary surface on the chassis made in Canada. While it is quite easy to see how the properties defined by the base SI units can be calibrated to ensure traceability, this is vastly more complicated for biological measurements. Ground truth for even a relatively simple measurement, such as the level of a surface marker on a particular type of immune cell, is not trivial to define. Also, the stability of biological materials is less consistent and predictable than that of chemical or physical ones.

Yet there is substantial value in biological reference or calibration materials. They can help tease out technical and biological variation in biological measurements and can be used to compare results from orthogonal approaches. As attested in the pages of this journal, reference materials can provide a benchmark for comparisons of methods and tools, sometimes in an interlaboratory format. Simple known mixtures of proteins or protein complexes, for instance, can help one gauge the performance of mass spectrometric pipelines. Fluorophore-labeled molecular rulers built on DNA origami can be used to compare the resolving power of microscopes and nanoscopes.

These are relatively early days for the development of widespread, standardized biological reference materials distributed by national metrological institutes. NIST, via the Genome in a Bottle Consortium, provides five well-characterized human genomes that laboratories can use as reference materials to check the quality of DNA sequencing; these activities are intended to extend to benchmarking analytical approaches like variant calling. The vanguard of applying metrological principles to biology appears to be in quality assurance of biologically based products, such as in clinical genomics, diagnostics and cell therapy, but such materials could be productively applied in research contexts as well.

To determine which areas would benefit most from a metrological approach and how to implement this, and to design reference materials, the first step is communication between biologists and measurement scientists. There is little to be lost, and much to be gained, through increased exchange between these groups. But perhaps more important, metrological principles can help bring a biologist's attention back to the fundamentals of experimental practice, even as techniques get ever more sophisticated: how to measure and record accurately so that one's conclusions about a system are as close as possible to reality.

Published online: 31 May 2018 https://doi.org/10.1038/s41592-018-0035-X 